\title{
CELUI QUE, CELUI DE, LE RETRANSLATIF EN QUESTION
}

\author{
Mora Millán, $\mathbf{M}^{\mathrm{a}}$ Luisa \\ Universidad de Cádiz. Facultad de Filosofia y Letras \\ Avda Gómez Llla sn, tlfno 956015521 , e-mail: Iuisa.moraaucaes \\ (Recibido, junio 2001; aceptado, julio 2001) \\ BIBLID [1133-682X (2000-2001) 8-9; 215-226]
}

\section{Resumen}

El estatuto gramatical que se atribuye a la unidad celui en contesto relativo asi como preposicional es ambiguo puesto que, a veces, es analizado como pronombre y otras como deteminante. En este articulo nos proponemos analizar dicho estatuto gramatical dentro del marco que nos ofrece la teoria de la trasposición de Bally y de la traslacion de Tesniere. En primer lugar hemos pretendido responder a la pregunta de si cs posible acordar una sola deseripeion sintactica. para contextos preposicionales y contextos relativos. En segundo lugar, hemos centrado nuestro análisis en los conceptos retranslatif, retranslation de Tesnière ya que éstos nos pemiten replantear la clave del problema.

Palabras clave: Bally, lesnicrc, rctraslativo, retraslacion, celut

\begin{abstract}
This paper intends to describe the syntactic comportement of celu units in prepositional and relative contexts. Traditionnally two syntactic analyses are proposed; pronom or article. We have tried to present the celutsyntactic comportement in Ch. Bally transposition theorie and L. Tesniere translation theorie. Later on we have analy sed retranslatif and retranslation concepts in $\mathrm{L}$. Tesnière because, we think, that perspective of syntactic analyse have to be changed in order to be able to explain cehu comporiement.
\end{abstract}

Key words: Bally, Tesniere, retranslatif. retranslation, celui.

\section{Résumé}

Le statut grammatical que l'on accorde à l'unité celu en contexte relatif et en contexte prépositionel est ambigu. Lantôt on fait appel à la classe pronominale tantôt à la classe des déteminants. Dans cel article nous nous proposons danalyser le statut accordé à l"unité celui à la lumière de la théorie de la translation de $\mathrm{L}$. Tesnière et de $\mathrm{C}$. Bally son precurseur. Nous nous sommes proposés. en tout premier lieu, d"essayer de savoir si la description des formes simples doit être unifiée. Par la suite. nous analyserons les concepts retranslatf, retranslatıon de $L$. Tesniere tout en signalant que c"est peut-être dans une demarche inverse, dans la mise en question de ce concept appliqué au suntagme prépositionnel. relatif. que le statut de celui peut être décélé.

Mots clés: Bally. Tesnière, retranslation, retranslatif, celui 


\section{Sumario}

0 . Introducción: But de l'article, 1. La description des formes simples (cehin qu-celư de) doit-elle être unifice?'1.1 Ch. Bally 1.2. L. Tesnière. 2. Le coneept de retranslation: Lne retranslation qui ne devalt pas avoir lieu.

\section{Introduction: But de l'article}

Le statut grammatical que l'on accorde à l'unité celul en contexte relatif el cn contexte prépositionel est ambigï, car tantôt on fait appel à la classe pronominale tantôt à la classe des déterminants. C'est le statut pronominal qui est le plus souvent répandu non seulement chez les grammairiens qui se placent dans une optique traditionnelle (tel est le cas de Grevisse 1936) mais aussi chez certains linguistes qui s'inscrivent dans une optique plus moderne (cf.Veland 1997). Quant au statut de déterminant, il faut dire qu'il a toujours $\mathrm{cu}$ ses défenseurs. Citons l'exemple classique ${ }^{1}$, et innovateur en mc̀me tomps, de la Grammaire de Damourettc \& Pinchon ou, plus actuellement, le cas de la psychomécanique (cfr. Pierrard 1991) qui élabore une présentation des différentes types d'études.

Dans cet article nous nous proposons d'analyser le statut accordé à l'unité celui à la lumière de la théorie de la translation de $\mathrm{L}$. Tesnic̀re et de $\mathrm{Ch}$. Bally. son précurseur. Notre étude envisage l'analyse des formes simples des pronoms démonstratifs ${ }^{2}$. c'està-dire des structures celui en contexte prépositionnel ct celui en contexte relatif, face aux formes composées celui-cli celui-là. La particularité que ces formes simplcs présentent à la lumière de la théoric de la translation est justement celle de permettre une interprétation ambiguë qui regrouperait le double statut à la fois pronominal et déterminant. Cela est-il possible?

Dans cet article, nous nous sommes proposé en tout premier lieu de vérifier si la description des formes simples doit être unifiée en présentant, par ordre chronologique. les solutions adoptées par Ch. Bally et $\mathrm{L}$. Tesnière qui mettent en relicf la complexité de ce problème syntaxique. Nous essaierons aussi de constater les ressemblances ed ou les différences qui existent entre cette théorie et celle de la transposition de Ch. Bally, apparemment semblables car, finalement, les deux analyses représentent deux points de vues fort différents. Tesmière.

Par la suite, nousaborderons l'analyse des concepts retranslatif, retranslation de L.

1. La description des formes simples (celui qu- / celui de) doit-elle être unifiée?

\subsection{Ch. Bally}

Ch. Bally semble répondre négativement à cettc question tout en postulant, dans son ouvrage Linguistique Générale et Linguistique Française, une description asymétrique des unités celui en contcxte relatif et en contexte prépositionnel. Effectivement, une description unifiée ne saurait, en principe, avoir de sens car s'il

1 Tn employant le terme classique non pas dans une acception scolastique car cette grammaire a toujours adopte un point de vue trés particulier et innovateur, comme on peut le constater dans la teminologite adoptée

2 Nous conservons la terminologie de L. Tesnière qui parle de pronoms et non pas de déterminants. 
est vrai qu'il affirme que le pronom celui, celle supplée l'article dans Le chapeau de Pierre et celui de Paul (Bally 1932:178) il est aussi vrai qu'il affirme, lors de l'actualisation explicite des termes virtuels (Bally 1932:88) l'équivalence entre L'oiseat quej'entends chanter et Celui qui règne dans les cieux.

Des pronoms se suppléent avec des valeurs identiques, p. ex. se et li dans «Paul s'admire el ne pense qu'à lui», que el quoi dans «le ne sais que faire ni à quoi me résoudre». Le pronom celui, celle supplée l'article dans «lc chapcau de Picrre ct celui do Pauln (Bally 1932:178)

D'après ces affirmations on a bien deux réalités différentes car d'une part, en contexte prépositionnel, on trouve la supplétion cehi + Déterminant et d'autre, en contexte relatif, on trouve la supplétion celui $+\mathrm{SN}$ ce qui aboutit à deux interprétations de lunité celui, considérée soit comme un déterminant $(l e)^{3}$, soit comme un syntagme (l'oiseau).

Pourtant il reste à spécifier le concept de supplétion. Pour Bally la supplétion consiste en l'emploi des signifiants hétérogènes qui ont exactement la même signification

Les signes supplétifs ont cxactement la méme signification, et des signifiants hétérogènes dont le rôle est déterminć par un choix arbitraire. (Bally 1032:178)

La supplétion ainsi conçue semble relever du domaine de la sémantique et correspondre avec le sens des mots ${ }^{4}$. Mais le contexte prépositionnel connait aussil l'actualisation explicite d'un terme virtuel. A ce propos Bally affirme

dans uce chapeau est celui de mon ami» ou "celui que j"ai acheté hier" celui équivaut à le chapeau... (Bally 1932:86)

L'interpétation syntagmatique (SN)
(a) Le chapeau de Pierre el
celui
de Paul
le chapean de Paul

se substitue donc, chez Bally, à l'interprétation déterminative
(b) Le chapean de Pierre et
celni
$L E$
de Paul
de Panl

3 Le terme déterminant est le produit de notre interpretation. Ch. Bally parle toujours de pronom lorsqu'il fait référence à l'unité cehi. Pourtant nous concluons que cela obéit à un usage spontanné du terme et qui reflète le poids de tout une tradition grammaticale mais qui n'est, en aucun cas, tout a fait en accord avec ses réllexions posterieures.

4 «Ainsi plusieurs radicaux de sens identique sont répartis entre les diftërentes formes d un mème verbe: fr allons. j'irai, va, je suis, je seral, jétats ..... (Bally 1932:1789. Une question sémantique qui dépasse le cadre de cet article est cette notion de sensidentique.

Pragmalingitistica, 8-9,2000-2001, 215-226 
On pourrait conclure que l'interprétation de l'unitć celui est colle d'un vcritable substitut nominal, c'est-à-dire d"un pronom aussi bien dans le contexte prépositionnel que dans le contexte relatif.

Quel sens faut-il accorder à son affirmation le pronom celui supplée l'article pour laquelle on pourrait penser à un glissement de l'unité celui de la classe des pronoms vers la classe des déterminants et à une interprétation donc déterminative?. Loin de là, la solution que Ch. Bally nous offre implique l'existence d'une ellypse textuelle, explication qui semble être précurseure de la description unifiée des subordonnées relatives des grammairiens générativistes. C'est ainsi que Ch.Bally explicitera à propos de celui de mon ami:

il (celui) introduit donc, au moyen de l'article le, qu'il contient implicitement, le virtucl chapean dans un nouveau actuel (de mon ami) (Bally 1932:86) ${ }^{6}$

Si le système de Bally nous offrait apparemment deux solutions syntaxiques différentes de l'unité celui en contexte prépositionnel et en contexte relatif, ill n'cn est pas ainsi tel que nous avons essayé de le démontrer. La description de $\mathrm{Ch}$. Bally permel d'accorder une solution syntaxique unifice et une telle solution semble constituer le germe de la description générativiste.

\subsection{Tesnière}

Contrairement à Bally, chez qui on n'a trouvé aucune analyse explicite des unités en contextc própositionnel/relatif, $L$. Tcsnicrc affirme ouvertement Ic parallèlisme entre les deux types de structures. Il manifeste le parallélisme cxistant entre le contexte prépositionmel qu'il dénomine la translation étale «Celui de Bernard» ct le contexte relatif, ćgalcment appelé, la translation descendante "Celui que vous m'ovez donné» (Tesnière 1959:479).

C'cst L. Tesnière qui, à l'intérieur de son système de la translation, nous offre des arguments syntaxiques et qui appuient le statut de déterminant de celui en contexte prépositionnel et relatif en accordant à l'unité celui le statut de retranslatif.

Qu'entend-il par retranslatif? Selon les mots de L. Tesnière un retranslatif est un élément qui a, par définition, toujours pour transférend un élément déjà transféré el n'a donc jamais à "opérer la translation que sur un élément qui en a déjà subi une première») (Tesnière 1959:479).

Fait pertinent de cette définition pour l'identification d'un retranslatif la restriction de son champ opératoire car autrement il faudrait considérer un comme un retranslatif non pas dans an livre noir et un rouge (translation simple) mais dans un livre de Plerre ef un d'Alfred (translation double). Pourtant, il n’est pas considéré comme un retranslatif par L. Tesnière car

5 Rappelons que, selon Bally (1965:88) dans les RSA il existe un parallelisme entre Qu perd gagne et Celus qu perd gagne, celui étant le concept virtuel à déteminer au moyen de la proposition subordonnée relative (cfr Mora Millan 1997).

6 C'est nous qui soulignons cette affimation «contenir implicitement» car, d'après nous, elle fait référence à la survivance du déterminant le dans la forme celu (explication diachronique des deux paradigmes CIST (cfr. Latin ECCE ISTE) et CLL (cfr. Latin ECCE ILLE)) mais elle fait surtout référence à une prise en compte des phénomènes grammaticaux dans une double optique, et syntaxique et sémantique, qui evoque pour nous la structure profonde et structure superficielle des générativistes. 
un peut s'appliquer dans les translations simples c'est pourquoi il est un translatif tout simplement. La classe des retranslatifs est ainsi réduite à celhi en contexte própositionnel (translatif du premier degré) ou celui en contexte relatif (translatif du second degré).

D'après l'appartenance de l'unité celu à la classe des retranslatifs, il faudrait reconnaître à cette unité le statut de mot vide. c est pourquoi Tesnière lui accorde le statul de déterminant. Tesnière va même plus loin car il appuie son analyse syntaxique sur une syntaxe comparée d’autres langues, notamment l’anglais

My father's book and Peler's one

et l'espagnol

El libro de Pedro y el de mi padre.

Mais l'on constate que dans ces deux langues les analyses syntaxiques revèlent deux réalités différentes car s il est vrai que el est considéré en espagnol comme un article et ne pourrait pas ètre autrement ${ }^{7}$ le cas de l'anglais cst fort différent car one se trouve être un pronom.

Pourtant, en contexte relatif. Iinterprétation déterminative de celu ne saurait si bien tenir car Tesnière reconnaît à toute proposition subordonnée relative le statut d'adjectir ce qui nous mène conséquemment à attribuer à celui le ròle d'antécédent (pronominal). Effectivement. dans l'cnoncé la jemme que $j$ 'ai vu peindre (p.581,stemma349) la proposition subordonnée relative est translatee en adjectif de discours, au moven de la composante translative du pronom relatif

$$
\begin{aligned}
& \text { Femme } \\
& \text { La } \\
& \begin{array}{c}
\text { qu-jai vu peindre } \\
J \quad \text { peindre } \\
\text {-e }
\end{array}
\end{aligned}
$$

Dans l'enoncé Ceux que nous avons vus germer (p.581. slemma352) Tesnière propose

$$
\operatorname{couxl}_{\substack{\text { I qu-avons vus } \\ \text { Nous germer } \\ \text {-e }}}^{\mathrm{A}}
$$

On voit mal comment justificr la première translation A de que nous avons vus germer si ce n'est qu'en considérant dans un premier stade ceux comme liunité antécédent;

7 Car, si la de Pedro. los de Pedro. las de Pedro permmetrait de penser au paradigme des pronoms, il nest pas ainsi dans le cas de el qui. lui, ne peut être qu'un article. 
Si on était obligé de reconnaître un ceux antécédent il serait de la sorte un mot plein ct non pas un mot vide ce qui lui enleverait son statut de retranslatif;

L'anaphorème du relatif reprend cette unité qui est reconnue forcément mot plein car elle possède une fonction à l'intérieur de la subordonnée relative. Tesnière distingue clairement entre ces deux types d'éléments:les indice (Les livres) et les anaphorique (Je les vois). Ces derniers sont des mots vides qui deviennent mots pleins dès que, dans une phrase, ils entrent en connexion anaphorique ovec un autre mot (p.86). En ce sens l'élément E/I anaphorème du pronom relatif est, lui aussi, un mot plein et devrait conséquemment, d'après nous, obéir à la même hiérarchie structurale et qui accorde au mot avec lequel les anaphoriques entrent en connexion un statut supérieur: celui, donc, d'antécédent.

Cela nous amène à proposer un stemma alternatif au stemma 352 et qui tout en étant équivalent du stemma 349 nous aiderait à comprendre la description unifiće, affirmée par Tesnière, de toutes les propositions relatives.

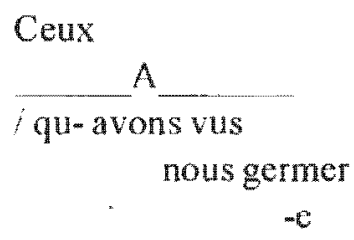

Tesnière affirme:

(1) l'existence d'une description unifée, comme déterminant, aussi bien en contexte prépositionnel qu'en contcxtc relatif.

(2) Mais on voit mal cette unification syntaxique du moment où il accorde cxplicitement aux relatives à emploi absolu (celui qui) le statut d'adjectif de discours. L'appartenance de ces relatives aux relatives adjectives semble contredire le statut de retranslatif de celui. A moins que l'on fasse intervenir une interprétation ellyptique qui permettrait à celui de conserver son statut de retranslatif (mot vide) et qui serait d'une part le déterminant (translatif) d"un Nom effacé qui permettrait l'adjectivation de la subordonnée relative; et d'autre, le dćtcrminant (retranslatif) qui permettrait le passage de cet adjectif de discours à substantif.

De cette façon la description accordée à ces unités par Ch. Bally ct par $\mathrm{L}$. Tesnière se rejoignent tout en permettant une solution syntaxique unifiée qui semble constituer le germe de la description générativiste qui se fonde dans la distinction structure superficielle / structure profonde.

\section{Le concept de retranslation: Une retranslation qui ne devrait pas avoir lieu.}

On a constaté qu'aussi bien C. Bally que L. Tesnière, malgré le parallélisme théorique affirmé entre celui et l'article, ils l'établissent dans la pratique entre celui et le syntagme nominal. Nous voilà devant une impasse syntaxique, qui a déjà constitué l'objet de longues réflexions (cl. Pierrard), car l'on sait bien les contraintes qui pèsent sur ce mot pour pouvoir être classé aussi bien dans la classe des déterminants quc dans la classe des pronoms. En restant fidèles à l'idée que celui supplée l'article du point de vue syntaxique, on aurait 
affaire au nouvel énoncé celui live d'Alfred et non pas celui d'Alfred auquel cas la supplétion qui a été élaborée concernerait le syntagme nominal complet le livre

Le noeud du problème se trouve, à notre avis, dans le problème posé par l'absorption du nom élidé et qui doit donc inverser l' optique sous laquelle le sujet a été traditionnellement étudié. Peut-on considérer d'Alfred/ que nous avons vus germer de véritables substantifs de discours? On obtient deux interprétations différentes possibles:

\section{Le/(live) d'Alfred}

Pour ceux qui se penchent vers une telle interprétation la réponse à la question est positive. Cette lecture implique l'aceptation de l'existence d'une retranslation du substantif Alfred, translaté en adjectif de discours d'Alfred, en un nouveau substantif de discours au moyen de retranslatif celui. Cette interprétation correspondrait à une interprétation déterminative de celui, car d'Alfred agirait comme un vćritable substantif dans le discours, auquel cas le nom élidé présenterait la formule Le / (livre) d'Alfred".

\section{Le (live) de Pierre}

Pour ceux qui, contrairement, penchent pour cette interprétation $B$ la réponse à la question, de ce point de vue, devient négative en ce sens où la retranslation n'a plus lieu. On trouve dans ce cas deux analyses syntaxiques cohérentes, qui font de l'unité en qucstion:

soit un pronom antécédent car ce qui, en principe, n'est qu'un déterminant prend, en contexte, un sens prégrant $t^{10}$.

soit un déterminant d'un nom elliptique, celui à déterminer au moyen des syntagmes prépositionnel et/ou relatif ${ }^{1}$

N'est-ce peul-ètre la translation double qui est en jeu? Il est bien évident que Alfred, nom, est translaté au moyen du translatif de en adjectif de discours (translation simple), mais il n'est pas si évident que le syntagme d'Alfred soit substantivé (translation double) car (nous inversons le raisonnement traditionnel) s'il était substantivé, pourquoi l'emploi du déterminant lui serait interdit (*le d'Alfred)?

Il bien vrai qu'il existe une dissymétrie dans lc paradigmc des déterminants $u \mathrm{~m} / \mathrm{le}$. Effectivement l'emploi de $u n$, translatif simple, dans la translation double (?) esi permis

8 C'est d'ailleurs l'opinion de F.Corbin (1990) pour qui les syntagmes en CELUI possèdent les caractéristiques syntaxiques et sémantiques générales d'un DSN.

9 C'est le point de vue que L. Tesnière semble adopter car c'est bien lui qui àté le premier à parler de concepts de retranslation et retranslatif.

10 C'est le même sens prégnant qui fait de la préposition avec un adverbe dans les emplois elliptiques du type Va jower avec!.

11 Remarquer que cette option diflère de la précédente car, si toutes les deux considèrent celu comme un déterminant, l'une accorde aux syntagmes prépositionne/ relatifle statut de substantif tandis que l'aulre leur accorde le statut d'adjectif. 
un livre de Pierre et un d'Alfred

tandis que le translatif simple le ne l'est pas.

*le livre de Pierve et le d'Alfred

Pourtant, il est bien évident que l'occurrence $a n d ' A l f r e d$ n'est correcte que dans des emplois où nous pourons restituer le nom elidé soit par le co-texte, soit le contexte: mais. en dehors de tels cas, un énoncé du type

* Un d Alfred est sur la table

deviendrait agrammatical.

Ce n'est que lorsque la substantivation se concrétise au niveau morphologique que l'emploi d'un déterminant (défini et/on indéfini) cst possible; et dans de tcls cas. la substantivation a. d'après nous, vraiment lieu car l'interprétation ellyptique n'est plus permisc:

\section{Ex. Le qu'en dira-ton}

le qui-vive

La solution donc. nous semble-t-il. il faut aller la chercher du côté des définitions mêmes des parties du discours car celles-ci ne sont pas que de simples unités syntaxiques mais en outre elles possèdent un composant sémantique dont il faut tenir compte dans l'analyse syntaxique 2 . C'est aussi en vertu du composant sémantique, et non simplcment syntaxique, que l'on a affaire dans le discours à la distinction nom vs adjectir. Dans l'énoncé J'aime le beau nous appréhendons beau de manic̀rc autonome, en tant que mol qui n'incide pas sur un autre mot. Le beau est ainsi compris au sens, autonome, de la beahé (comme un substantif). II n'cn est pas de mème pour Le livre noir el le rouge où rouge n'est pas autonome mais se trouve appliqué au nom hrve (il est donc un adjectif). Cette incidence de rouge à livre et qui a lieu indiscutablement au niveau sémantique doit aussi être traduite au nireau syntaxique, au moyen d'une interprétation ellyptique.

\section{J'aime le rouge, c'est ma conleur préférée (substantit) \\ Est-ce que th aimes le chapeau vert que je vais m'acheter? Non j'aime mieux le ronge (emploi déictique > interpretation elliptique le (livre) rouge) (adjectir)}

Or, la substantivation des syntagmes prćpositionnels qui a lieu, d’après L. Tesnière. dans la translation double à retranslatif celu doit être questionnée aussi bien syntaxiquement que sćmantiquement car. le syntagme prépositionnel, tout en étant un adjectif, il ne faut pas oublier qu'il est un adjectif de discours et, pourtant, qu'il est loin de posséder les mêmes caractéristiques syntactico-sémantiques qu*un simple adjectif. Un tel syntagme

12 Cf Coseriu (1978) ou il definit les catégories verbales comme des modes de signifer les mots dans le discours. 
prépositionnel ne peut pas commuter avec une fonction nominale. dans d'autres contextes. C'est la raison pour laquelle la substantivation n "est pas possible en dehors des emplois en mention (Ex. "De mon ami" est un syntagme prépositionnel). Un tel syntagme prépositionnel, en tant qu'adjectif de discours, est toujours incident à un terme nominal. D'où nous concluons qu' il existe bien une translation simple dans

Lelive d'Alfred

qui fait de Alfred (substantif), un adjectif de discours, au moyen du translatif de (mot vide). Mais qu'il n'existe pas de retranslation du syntagme prépositionnel d'Alfred cn substantif, au moyen du retranslatif cehi, car le syntagme prépositionnel d'Alfred n'est pas un substantif, même de discours. mais qu il reste un adjectif (l' esprit le conçoit comme incident à un autre terme et non pas de manière autonome ${ }^{13}$ ). En considérant celle possibilité d'inexistence du processus retranslatif celui cesserait d'être un retranslatif (mot vide) pour devenir un véritable mot plein. avec ce sens prégnant auquel nous avons fait mention, et permettre ainsi sa détermination au moyen d un syntagme prépositionnel et /ou relatif.

De même qu'il arrivait dans l'analyse des RSA proposée par L. Tesnière, car elles étaient considérées comme des relatives adjectives et non pas comme des substantives. Nous étions convemus dans Mora Millán (1997:41) que le processus de retranslation des subordonnées adjectives en substanives, au moyen du retranslatif celui, n'ćtait pas si ćvident. Les RSA sont toujours senties comme des subordonnées adjectives appliquées à un substantif omis.

S'il en était ainsi. l'on pourrait expliquer certains compontements syntaxiques. A savoir, la possibilité de trouver un adjectif en apposition après celui. C'est-à-dire la possibilité (tendance) de placer un adjectif en apposition dans le chapeau noweau de Pierre et cehi de Paul, incidant sur celui et non pas sur de Paul (le chapeau nowvean de Pierre et celm vieux de Paul $>$ celui vieuxide Paul vs * celuivieux de Pauls.

On en trouve l'exemple suivant chez. Grevisse

Ils passatent la, chaque jour, une heure bénie qu'ils avaient l'impression d'arracher a toutes les tyranies conjures: celle. farouche de l'argent. et celle, caressante et souveraine, du foyer (G.Duhamel Deux hommes p. 131).

A notre avis, cet adjectif en apposition ne serait pas une structure syntaxiquement correcte si linterprétation de celui était celle de mot vide ou déterminant comme le prouve l'agrammaticalité de l'énoncé (car il ne s'agit pas là d'une simple antéposition de l'adjectif. ce qui serait tout à fait correct).

13 Il cxiste toujours dans ces syntagmes prépositionnels soit des rapports de complementation nominale (exprimant la possession: possesseur. chose possédée) soit des rapports appositifs (la ville de Paris et celle de Madrid).

14 Cr Koch Krefeld (1995:241) qui parlent du potenticl connexionnel supericur el interieur comme un facteur décisif dan la définition du concept de translation cla translation implique une différence connexionnelle entre le transféré el le transtërende qui, par définition. garde son potentrel connexonnel inferen"w. 
*ce, vieil, homme

La structure CELUI, ADJ, SP / REL est, donc, assez répandue:

De Tchernobyl au Danube, elles savent ce qu'il en coute d'agresser la nature ou de la délaisser L'enjeu, vous le connaissez. C'est celui du rapporl quotidien de l'homme à son cadre de vie comme cehi, millénaire, de la société à la nature (J.Delors Le nonveau concert européen)

Ón pourrait aussi constater la possibilité de trouver un complément du nom derrière celui. Un déterminant ne peut, en aucun cas, être restreint au moyen d'un complément du nom. C'est ainsi que l'on peut dire

Ce regroupement syntaxique nous semble s'imposer autant que "cehi des mots" qui suivent le verbe être

et non pas

Ce regroupement syntaxique nous semble s'imposer autant que "le des mots" qui suivent le verbe être

Nous concluons que le syntagme préposilionnel/ relatif, adjectif de discours, ne peut pas être retranslaté en substantif, et c'est là que l'existence d'un celui retranslatif se trouve. elle même, mise en question.

D'après ce que l'on constate dans les emplois recensés, de par leur comportement syntaxique c'est l'unité celui, au détriment du syntagme prépositionnel/ relatif, qui a toutes le chances d'être substantivée. Cf. Tesnière (1959:479):

Une des conséquences de la valeur translative substantivale de aceluin ct de ses équivalents étrangers, $e^{\prime}$ 'est la tendance qu'ont ces mots à prendre l'article, qui est le translatif substantival [ ....] la chose est manifeste en français populaire, les patois et en particulicr en Normandie «La ferme de Pierre Bénédicte?... C'est la troisième après la cclle à Porée" (Maupassant, Contes, L'abandonné).

D'après cette affirmation de Tesnière, la supplétion, dont nous parlions tout au début du texte, ne concerne pas l'article mais le substantif lui même «celui supplće non pas l'article mais le substantif mêmè. D'où le parallèlisme entre

La ferme de Pierre Bénédicte

La celle à Porée

auquel cas celui ne serait pas un retranslatif mais un translaté. 


\section{Refferences}

BALLY, C. (1932) Le langage et la vie. Francke, Berne, 1952.

BALLY, CH.(1965) Linguistique générale el linguistique française. Francke, Berne.

CORBIN, F. (1990) «Les groupes nominaux sans nom du français» in Kleiber G. Tyvaert J.E. (dir) L'anaphore et ses domaines. Recherches linguistiques de Metz, 14, 6380.

COSERIU, E. (1978) "Sobre las categorías verbales" dans Grammática, Semántica, Universales. Madrid, Gredos.

GREVISSE, M. (1936) Le bon usage. Paris-Bruxelles, Duculot 1980

MORA MILLÁN, ML. (1997) "Transposition, translation et subordonnées relatives" Revista de Filología Francesa. N112. Homenaje al Profesor D. Jesús Cantera Ortíz de Urbina. Servicio de publicaciones de la Univ. Complutense, Madrid 1997, 35-42.

PIERRARD, M. (1991) "Observations sur le statut syntaxique et sémantique de celui + relative" Trowaux de Linguistique 1991, 22, 69-91.

TESNIERE, L. (1959) Eléments de syntaxe structurale. Paris, Klincksicck, 1976.

VELAND, R. (1997) "Cet adjectif qui ne simule pas: le problème de la détermination du pronom CELUT" Le Français Moderne. LXV, 169-183. 